\title{
Detection of Planar Regions in Volume Data for Topology Optimization
}

\author{
Ulrich Bauer and Konrad Polthier \\ FU Berlin, \\ Arnimallee 3, 14195 Berlin, Germany \\ \{ubauer, polthier\}@mi.fu-berlin.de \\ http://geom.mi.fu-berlin.de/
}

\begin{abstract}
We propose a method to identify planar regions in volume data using a specialized version of the discrete Radon transform operating on a structured or unstructured grid. The algorithm uses an efficient discretization scheme for the parameter space to obtain a running time of $\mathcal{O}(N(T \log T))$, where $T$ is the number of cells and $N$ is the number of plane normals in the discretized parameter space.

We apply our algorithm in an industrial setting and perform experiments with real-world data generated by topology optimization algorithms, where the planar regions represent portions of a mechanical part that can be built using steel plate.
\end{abstract}

Keywords: Discrete Radon transform, Hough transform, Plane detection, Topology optimization.

\section{Introduction}

The field of topology optimization studies the automatic generation of mechanical parts with an a priori unknown topological shape [1]. Prominent techniques include continuous methods [12]3, which optimize a 3D density function in the given work space, or truss methods [4/5, which optimize and rearrange a construction of stiff linear elements connected at junctions points. In the present paper we analyze data arising from continuous methods. Since these methods often produce output defined on unstructured grids such as tetrahedral meshes, our method is designed to work on this kind of data.

The density function obtained from continuous methods is a coarse model of the optimal structural design. Typically, post processing of the density function is needed in order to obtain a constructible shape. For example, planar metal plates are a simple and cheap building block to physically realize a mechanical part. We present an algorithm which automatically computes a set of planar regions which best approximates a given density function arising from topology optimization.

By a planar region, we denote a connected component of the intersection of some plane with the support of the given density function $\Omega \subset \mathbb{R}^{3}$, i.e. the domain on which the density function is defined. Our algorithm solves the problem

F. Chen and B. Jüttler (Eds.): GMP 2008, LNCS 4975, pp. 119-126 2008.

(C) Springer-Verlag Berlin Heidelberg 2008 


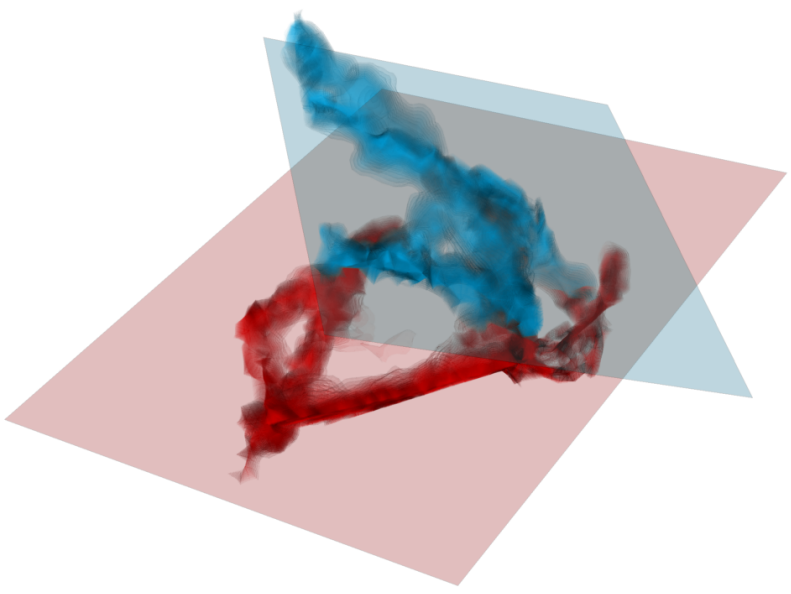

Fig. 1. A volume rendered density function and two planar regions, visualized by their corresponding planes. Volume data colored according to the closest planar region.

to find the planar region that covers most density. The process can be repeatedly applied after purging the density around the optimal planar region.

Our algorithm uses a discrete Radon transformation [6] and computes the integrated density of planar regions through the work space. Regions with highest integral density become candidates for the final construction (cf. Fig. 1). A similar technique for 2D images is known as Hough Transform [7/8. The space of planes in $\mathbb{R}^{3}$ is discretized using a triangulation of the unit sphere together with an optimized traversal algorithm to reduce the number of sorting procedures.

The algorithm grew out of an industry collaboration with ThyssenKrupp Tallent Ltd., and has been tested on various datasets from automotive industry. We provide experimental evidence of the reliability and efficiency of our implementation.

\subsection{Related Work}

The proposed technique can be considered as a discretization of a variant of the Radon transform in $\mathbb{R}^{3}$ on piecewise constant functions defined on structured or unstructured grids. The most well-known discretization of a Radon transform is the Hough Transform [78], which is defined on a 2-dimensional grid representing a pixel image.

Other discretizations of the Radon transform have been proposed in [9] for 3-dimensional regular grids, and in [10[11 for unstructured point clouds in $\mathbb{R}^{3}$. A common problem in these schemes is that the configuration space, in particular the space of undirected plane normals, namely the projective plane $\mathbb{R P}^{2}$, is represented by a single-chart parametrization over a subset of $\mathbb{R}^{2}$, and contains singularities where a piece of the boundary of the parameter domain is mapped onto a single point. In the vicinity of these singularities, unbounded metric distortion is unavoidable. Uniform sampling in the parameter domain therefore 
leads to drastic oversampling near the singularities. We propose a discretization that aims at equal distribution of sampling points on $\mathbb{R P}^{2}$. Note that this is not an issue for the usual Hough transform in $\mathbb{R}^{2}$, since the space of unoriented normals $\mathbb{R} \mathbb{P}^{1}$ can be parametrized without metric distortion.

A different scheme using clustering of local estimates for plane detection in point clouds has been proposed in [12. This approach, however, has disadvantages: first, the local estimation of best-fitting planes is susceptible to noise and requires an additional parameter determining the estimation range. Moreover, finding clusters is a complex problem. In comparison, discrete variants of the Hough transform require no local estimates and no initial values, are very robust to noise, and deterministically find the optimum in the discrete parameter space by exhaustive search.

Other examples (with a different scope) of post-processing methods with the goal to extract production-relevant data from data generated by structural optimization tools can be found in 1314 .

\subsection{Overview}

In Section 2, we review the problem in the smooth setting. Section 3 describes the proposed discretization of the problem. In Section 4, we provide a complexity analysis of the algorithm, and Section 5 contains results of our algorithm on test data.

\section{The Radon Transform and Generalizations}

Consider a density function $\rho: \Omega \subset \mathbb{R}^{3} \rightarrow \mathbb{R}$ with support on a compact domain $\Omega \subset \mathbb{R}^{3}$. The (generalized) Radon transform of $\rho$ is defined as the integral of $\rho$ over a hyperplane $H \in \mathbb{H}[\underline{6}$ :

$$
\mathcal{R}[\rho](H)=\int_{H} \rho(x) \mathrm{d} x
$$

In his original work, Radon's main interest was on the inverse Radon transform, which allows to reconstruct the function $\rho$ from the integrals over hyperplanes; this transform found a particularly important application in the evaluation of computer tomography data. But also the Radon transform gained interest in a particular discretization for 2D images, called the Hough Transform [78, which is an important tool for line detection. One notable property of the Hough transform (and the Radon transform in general) is its high robustness to noise, because only quantities integrated over large domains are considered.

Maxima of this function over the set of hyperplanes $\mathbb{H}$ correspond to planar features. To incorporate geometric locality into the search for planar regions, we can restrict the integration to connected components of the intersection of $H$ with the domain $\Omega$ and obtain the following optimization problem:

$$
\max _{\substack{D \subset \Omega \cap(H \in \mathbb{H}) \\ D \text { connected }}} \int_{D} \rho(x) \mathrm{d} x
$$


To smooth the density function and to allow a certain tolerance for off-plane deviation, we can enlarge the integration domain to include all points having distance at most $w$ from the hyperplane

$$
H_{w}=\{x: d(H, x) \leq w\},
$$

i.e. the intersection of two halfspaces, called fattened plane of width $w$, and consider the optimization problem

$$
\max _{\substack{D \subset \Omega \Omega H \\ D \text { connected }}} \int_{D} \rho(x) \mathrm{d} x
$$

This smoothing is of special interest in our application, since the density function computed by topology optimization algorithms is often concentrated in bone-like structures (cf. Figs. 1 and 3). To recognize planar regions formed by these "bones", one would like to set the width $w$ to approximately match the width of such a bone. This is especially important when the optimization process is applied iteratively, because the density covered by the fattened plane has to be purged after each iteration. If the width $w$ is chosen too small, some density in the vicinity of the optimal planar region is left and will be considered in the next iteration. As a consequence, several similar planar regions can be found, corresponding to the same feature in the input data. To avoid this problem, the parameter $w$ must be chosen large enough that the planar features are completely covered by the fattened planes.

\section{Detection of Planar Regions in Discrete Data}

Assume that we are given a structured or unstructured grid with a piecewise constant nonnegative scalar density function assigned to each cell. We are now searching for the fattened plane that covers most density. To simplify computation, we are using a lumped mass model, i.e. we assume that the whole mass inside a cell is concentrated at its barycenter. The mass of a cell is computed as the density multiplied by the volume of the cell. If the width $w$ of the fattened plane is considerably larger than the typical diameter of the cells, this simplification introduce only negligible artifacts.

\subsection{Discretization of the Parameter Space}

The described problem is a global nonlinear optimization problem, but it only has a 3-dimensional parameter space, which makes it feasible for exhaustive search in an appropriate discretization of the parameter space. We choose the following parametrization for the parameter space, which is the space of unoriented planes. We describe each plane by a normal vector $\boldsymbol{n}$ with $\|\boldsymbol{n}\|=1$ and a distance to the origin $d$. Since we are only considering unoriented planes, we also assume $\langle\boldsymbol{n},(1,0,0)\rangle \geq 0$. This means that the space of normals considered can be parametrized over a unit hemisphere. To obtain a discrete search space for the normals, we therefore are looking for an even distribution of points on the unit 


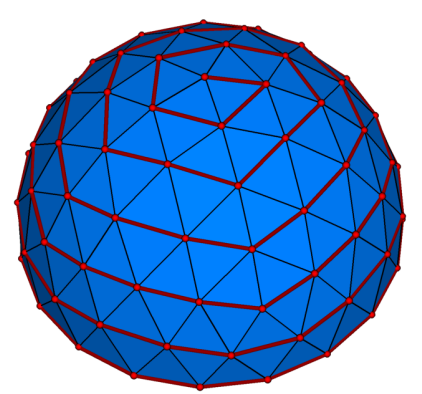

Fig. 2. A triangulated hemisphere, obtained by successive subdivision of the icosahedron, with a Hamiltonian path over the edge graph

(hemi-)sphere. This is achieved by repeated 1-4 subdivision of an icosahedron, each time followed by a projection of the new vertices onto the unit sphere (see Fig. (2). For a more in-depth discussion about the problem of distributing points evenly on the sphere, see [15].

To obtain a discretization for the range of distances $d$, we first move the origin to the centroid of the volume mesh. Now, for every direction $\boldsymbol{n}$, we compute the smallest range of distances $d$ containing all planes with normal $\boldsymbol{n}$ going through the data points, by sorting the data points with respect to their scalar product with the normal $d_{P}=\langle P, \boldsymbol{n}\rangle$. This range is then equidistantly discretized.

\subsection{Sweeping the Parameter Space}

With the data points sorted in direction of the normal $\boldsymbol{n}$, we can now easily slide a window $[d-w, d+w]$ over the range of distances. We use an accumulator variable to count the mass of all points in the range $[d-w, d+w]$ w.r.t. the normal $\boldsymbol{n}$. Each time we proceed to the next discrete value of $d$, we add to the accumulator the mass of all newly covered points, and subtract the mass of all points no longer covered by the window. Since the points are sorted, this can be done in constant time for each point.

If we are only searching for the mass in connected components inside the window (where connectivity is induced by the grid), we also remember the first and the last index of the (sorted) points covered by the current window. We then do a traversal of the connectivity graph by breadth first search to find all connected components and to compute their respective masses.

When we iterate through the discrete set of normals, we make sure that subsequent normals are joined by an edge of the subdivided icosahedron, and therefore do not vary much. This is ensured by ordering the vertices of the triangulated hemisphere by a Hamiltonian path over the edge graph. Such a Hamiltonian path can easily be found by "spiraling" from the north pole to the equator (see Fig. (2). The benefit of using this ordering of the normals is that complexity of two subsequent sorting operations is low when the two normals are very similar, because the two resulting sorting sequences are also similar. 


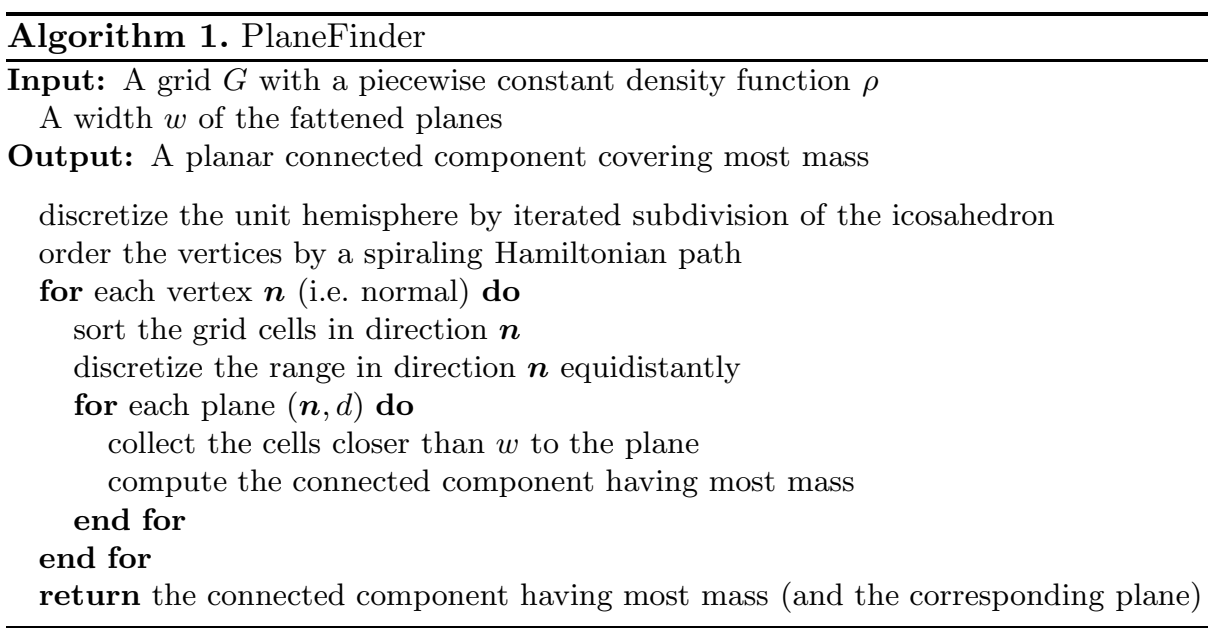

\subsection{Purging Dominant Planar Features}

After a complete sweep through the discretized parameter space, we have found an optimal plane with a corresponding set of grid cells. We now purge the density values on this set of cells. This allows to find further planar regions, avoiding the possibility that another optimum covers essentially the same data.

\section{Complexity Analysis}

Let $T$ denote the number of cells, $N$ be the number of discrete normal directions for the planes (the number of vertices of the triangulated hemisphere), and $D$ be the maximal number of planes checked in any direction. Assuming $D \in o(T)$ and $w \in \mathcal{O}\left(\frac{1}{D}\right)$, the total running time of the algorithm is $\mathcal{O}(N(T \log T))$.

For each normal, the set of lumped mass points is sorted, which is done in time $\mathcal{O}(T \log T))$. Then the set of points covered by the current plane is collected, taking time $\mathcal{O}(T)$ in total. For each of these sets, a breadth-first search is performed to compute the connected components of the induced subgraph of the cell connectivity graph. Each vertex is covered by a constant number of fattened planes, since $w \in \mathcal{O}\left(\frac{1}{D}\right)$. Moreover, since each vertex of this graph has degree at most 4, traversal of each of these graphs also takes $\mathcal{O}(T)$ in total.

\section{Results}

We tested our algorithm on real-world instances with between 150000 and 300000 tetrahedra. On a typical instance with 163799 tetrahedra, 43042 of which carried non-zero density, computation of the optimal plane took about 25 seconds on a standard laptop $(2.16 \mathrm{GHz}, 2 \mathrm{~GB}$ RAM). The dimensions of the 


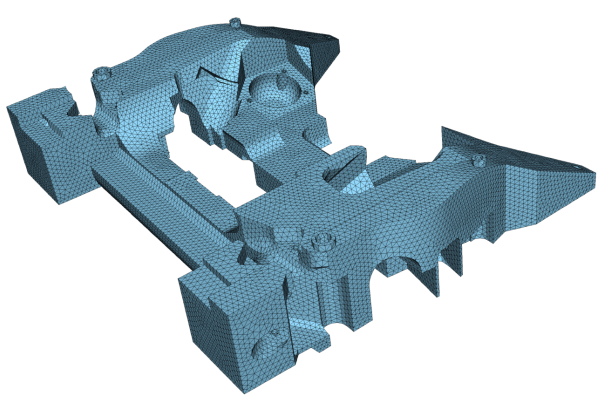

(a)

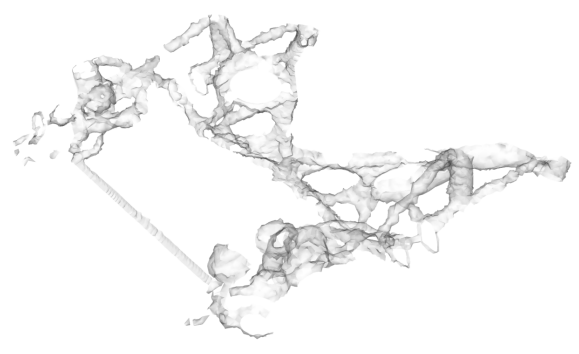

(c)

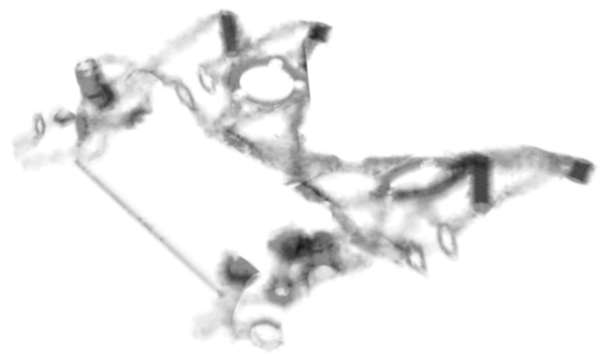

(b)

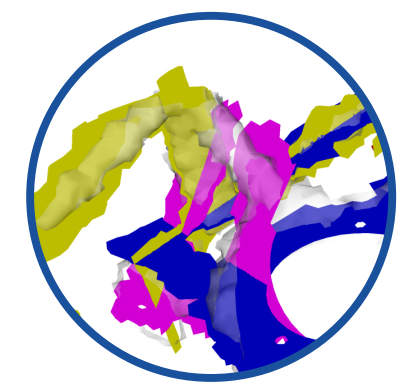

(e)

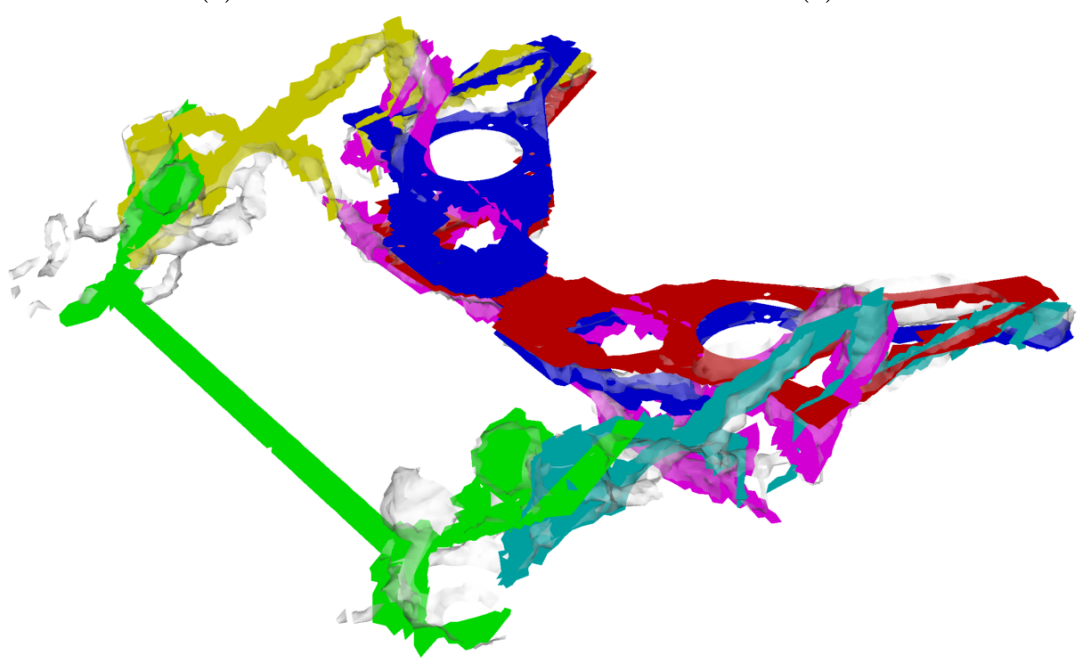

(d)

Fig. 3. Identifying planes on a typical density distribution generated with a topology optimization software. (a) The input domain. (b) A volume rendering of the density function. (c) An isosurface of the density function, shown transparent. (d) The 6 most dominant planar regions found using our algorithm. (e) A closeup of (d). 
bounding box of the tetrahedral mesh are approximately $80 \times 90 \times 20 \mathrm{~cm}$; the parameter $w$ was set to $2 \mathrm{~cm}$. The results computed using 6 iterations of the algorithm are visualized in Figure 3.

Acknowledgments. The work of Ulrich Bauer was supported by ThyssenKrupp Tallent Ltd., UK. Konrad Polthier is supported by the DFG Research Center MATHEON "Mathematics for key technologies" in Berlin. Thanks to Adrian Chapple from ThyssenKrupp Tallent for providing volume data.

\section{References}

1. Bendsøe, M.: Topology Optimization: Theory, Methods, and Applications. Springer, Heidelberg (2003)

2. Stolpe, M.: On Models and Methods for Global Optimization of Structural Topology. KTH, Mathematics, Stockholm (2003)

3. Sigmund, O.: A 99 line topology optimization code written in Matlab. Structural and Multidisciplinary Optimization 21(2), 120-127 (2001)

4. Ben-Tal, A., Bendsøe, M.: A New Method for Optimal Truss Topology Design. SIAM Journal on Optimization 3, 322 (1993)

5. de Klerk, E., Roos, C.,T.T.: Semi-definite problems in truss topology optimization. Technical Report Report 95-128, Faculty of Technical Mathematics and Informatics, Delft, Netherlands (1995)

6. Radon, J.: Über die Bestimmung von Funktionen durch ihre Integralwerte längs gewisser Mannigfaltigkeiten. Berichte Sächsische Akademie der Wissenschaften, Leipzig, Mathematisch-Physikalische Klasse 69, 262-277 (1917)

7. Hough, P.: Method and Means for Recognizing Complex Patterns, United States Patent US 3,069,654, 18.12.2002 (1962)

8. Duda, R.O., Hart, P.E.: Use of the Hough transformation to detect lines and curves in pictures. Commun. ACM 15(1), 11-15 (1972)

9. Sarti, A., Tubaro, S.: Detection and characterisation of planar fractures using a $3 \mathrm{~d}$ hough transform. Signal Processing 82(9), 1269-1282 (2002)

10. Vosselman, G., Dijkman, S.: 3D building model reconstruction from point clouds and ground plans. International Archives of Photogrammetry and Remote Sensing 34(3/W4), 37-43 (2001)

11. Kurdi, F.T., Landes, T., Grussenmeyer, P.: Hough-Transform and Extended RANSAC Algorithms for Automatic Detection of 3D Building Roof Planes from Lidar Data. In: Proceedings of the ISPRS Workshop on Laser Scanning, pp. 407-412 (2007)

12. Peternell, M., Steiner, T.: Reconstruction of piecewise planar objects from point clouds. Computer-Aided Design 36(4), 333-342 (2004)

13. Paralambros, P.Y., Chirehdast, M.: An integrated environment for structural configuration design. Journal of Engineering Design 1, 73-96 (1990)

14. Hornlein, H., Kocvara, M., Werner, R.: Material optimization: bridging the gap between conceptual and preliminary design. Aerospace Science and Technology 5(8), 541-554 (2001)

15. Katanforoush, A., Shahshahani, M.: Distributing Points on the Sphere, I. Experimental Mathematics 12(2), 199-209 (2003) 